\title{
Profissionais e futuros profissionais da voz: ambiente de trabalho, hábitos vocais
}

\section{incorretos e queixas vocais}

\author{
Voice professionals and future professionals: work environment, incorrect vocal habits and vocal \\ complaints
}

Profesionales de la voz y futuros profesionales: entorno laboral, hábitos vocales incorrectos y quejas vocales

Recebido: 31/01/2021 | Revisado: 07/02/2021 | Aceito: 19/02/2021 | Publicado: 27/02/2021

\author{
Gabriele Rodrigues Bastilha \\ ORCID: https://orcid.org/0000-0003-4207-8001 \\ Universidade Federal de Santa Maria, Brasil \\ E-mail: fonogabriele@gmail.com \\ Débora Bonesso Andriollo \\ ORCID: https://orcid.org/0000-0002-6461-180X \\ Universidade Federal de Santa Maria, Brasil \\ E-mail: deborabandriollo@gmail.com \\ Carla Aparecida Cielo \\ ORCID: https://orcid.org/0000-0002-7219-0427 \\ Universidade Federal de Santa Maria, Brasil \\ E-mail: cieloca@yahoo.com.br
}

\begin{abstract}
Resumo
Objetivo: Verificar e associar as características desfavoráveis à produção da voz no ambiente de trabalho, os hábitos vocais incorretos e as queixas vocais de profissionais e futuros profissionais da voz. Método: Estudo transversal, nãoexperimental, retrospectivo e quantitativo, com a utilização de banco de dados de uma clínica-escola de Fonoaudiologia. Foram selecionados 137 registros do banco de dados sobre profissionais e futuros profissionais da voz, adultos, do sexo masculino e feminino. Os dados extraídos dos registros relacionavam-se a fatores desfavoráveis à produção da voz no ambiente de trabalho, aos hábitos vocais incorretos e às queixas vocais. Foram utilizados o Teste de Hipótese para Proporção, Teste Qui-quadrado de Pearson e Teste Exato de Fisher com nível de significância de 5\%. Resultados: Houve frequência estatisticamente significativa de fatores desfavoráveis à produção da voz no ambiente de trabalho, hábitos vocais incorretos e queixas vocais; houve associação significativa entre fatores desfavoráveis à produção da voz no ambiente de trabalho e hábitos vocais incorretos, e entre fatores desfavoráveis à produção da voz no ambiente de trabalho e queixas vocais. Conclusão: Neste grupo de profissionais e futuros profissionais da voz, verificou-se alta ocorrência de fatores desfavoráveis à produção da voz no ambiente de trabalho, queixas vocais e hábitos vocais incorretos, e associação das queixas vocais e hábitos vocais incorretos com fatores desfavoráveis à produção da voz no ambiente de trabalho. Portanto, destaca-se que a prevenção, somada a melhores condições de trabalho, pode resultar em diminuição dos quadros de disfonia em profissionais e futuros profissionais da voz.
\end{abstract}

Palavras-chave: Ambiente de trabalho; Disfonia; Ergonomia; Saúde do trabalhador; Voz.

\begin{abstract}
Objective: To verify and associate the unfavorable characteristics of voice production in the workplace, incorrect vocal habits and vocal complaints from professionals and future voice professionals. Method: Cross-sectional, nonexperimental, retrospective and quantitative study, using a database from a speech therapy clinic-school. Were selected 137 records from the database about voice professionals and future voice professionals, male and female, adults. The data extracted from the records related to unfavorable factors of voice production in the workplace, incorrect vocal habits and vocal complaints. The Proportion Hypothesis Test, Pearson's Chi-square Test and Fisher's Exact Test used with a 5\% significance level. Results: There was a statistically significant frequency of unfavorable factors of voice production in the workplace, incorrect vocal habits and vocal complaints; there was a significant association between unfavorable factors of voice production in the workplace and incorrect vocal habits, and between unfavorable factors of voice production in the workplace and vocal complaints. Conclusion: In this group of professionals and future voice professionals, there was a high occurrence of unfavorable factors of voice production in the workplace, vocal complaints, and incorrect vocal habits, as well as an association of vocal complaints and incorrect vocal habits with unfavorable factors of voice production in the workplace. Therefore, it is noteworthy that prevention, associated with better working conditions, can result in a decrease in dysphonia in professionals and future voice professionals.
\end{abstract}


Keywords: Working environment; Dysphonia; Ergonomics; Occupational health; Voice.

\section{Resumen}

Objetivo: Verificar y asociar las características desfavorables del ambiente de trabajo, los hábitos vocales incorrectos y las quejas vocales de los profesionales y futuros profesionales de la voz. Método: Estudio transversal, no experimental, retrospectivo y cuantitativo, utilizando una base de datos de una clínica-escuela de logopedia. Se seleccionaron 137 registros de la base de datos sobre profesionales de la voz y futuros profesionales de la voz, adultos, hombres y mujeres. Los datos extraídos de los registros se relacionaron con factores desfavorables en el ambiente de trabajo, hábitos vocales incorrectos y quejas vocales. Se utilizó la Prueba de Hipótesis de Proporción, la Prueba Chicuadrado de Pearson y la Prueba Exacta de Fisher con un nivel de significancia del 5\%. Resultados: Hubo una frecuencia estadísticamente significativa de factores desfavorables en el ambiente de trabajo, hábitos vocales incorrectos y quejas vocales; hubo una asociación significativa entre factores desfavorables en el ambiente de trabajo y hábitos vocales incorrectos, y entre factores desfavorables en el ambiente de trabajo y quejas vocales. Conclusión: En este grupo de profesionales y futuros profesionales de la voz, hubo una alta ocurrencia de factores desfavorables en el ambiente de trabajo, quejas vocales, hábitos vocales incorrectos, así como una asociación de quejas vocales y hábitos vocales incorrectos con factores desfavorables en el ambiente de trabajo. Por tanto, es de destacar que la prevención, asociada a mejores condiciones laborales, puede resultar en una disminución de la disfonía en los profesionales y futuros profesionales de la voz.

Palabras clave: Ambiente de trabajo; Disfonía; Ergonomia; Salud laboral; Voz.

\section{Introdução}

A voz, essencial para a comunicação e relações interpessoais, também pode ser usada profissionalmente, sendo conhecida como voz profissional. Professores, atores, cantores, advogados, telefonistas, locutores e outros profissionais necessitam da voz para transmitir conhecimentos e sentimentos, bem como para enriquecer seu discurso (Andriollo, Bresolin, Frigo, \& Cielo, 2020; Cielo, Christmann, Ribeiro, Hoffmann, Padilha, Steidl \& Frigo, 2014; Cielo, Ribeiro \& Bastilha, 2015; Cielo, Ribeiro \& Hoffmann, 2015; Siqueira, Bastilha, Lima \& Cielo, 2016; Vilanova, Marques, Ribeiro, Oliveira, Teles \& Silverio 2016).

Principalmente nesses profissionais, os maus hábitos vocais são realizados frequentemente como, por exemplo, falar muito ao telefone, falar forte, em grande quantidade ou por muito tempo, gritar, dentre outros. Isto pode levar a um distúrbio vocal, sendo que os tecidos das pregas vocais podem ser danificados (Abou-Rafée, Zambon, Badaró, \& Behlau, 2018; Cielo, Ribeiro et al., 2015; Jesus, Ferrite, Araújo, \& Masson, 2020; Pinho, 2007; Siqueira, Bastilha et al., 2016). As condições de trabalho, ou seja, os aspectos do ambiente de trabalho que compreendem agentes de risco físicos, químicos e biológicos, quando em grande intensidade ou concentração, também podem influenciar a saúde vocal e geral do trabalhador (Abou-Rafée, Zambon et al., 2018; Branco, Silva, Jansen, \& Giusti, 2011; Cielo, Ribeiro et al., 2015; Ferreira, Akutsu, Luciano, \& Viviano, 2008; Jesus, Ferrite et al., 2020; Karakaya, Karakaya, Tunç, \& Kihtir, 2015; Kraemer, Moreira, \& Guimarães, 2020; Mango, Carilho, Drabovski, Joucoski, Garcia, \& Gomes, 2012; Vilanova, Marques, Ribeiro, Oliveira, Teles, \& Silverio, 2016).

Profissionais da voz têm tendência a apresentar mais problemas vocais do que outros profissionais e, além das queixas vocais, são comuns relatos de sintomas físicos de desconforto (Abou-Rafée, Zambon et al., 2018; Andriollo, Bresolin et al., 2020; Cielo, Christmann et al., 2014; Cielo, Ribeiro et al., 2015; Cielo, Ribeiro et al., 2015; Kraemer, Moreira et al., 2020). Essas queixas vocais e/ou corporais podem resultar da soma de maus hábitos vocais e condições de trabalho precárias ou inadequadas.

Recentemente houve o reconhecimento legal dos Distúrbios de Voz Relacionados ao Trabalho (DVRT), sendo classificados como "qualquer forma de desvio vocal relacionado à atividade profissional que diminua, comprometa ou impeça a atuação ou a comunicação do trabalhador, podendo ou não haver alteração orgânica da laringe" (Brasil, 2018). Tal marco é um avanço para os profissionais que dependem da voz e que agora contam com esse respaldo, considerando que os DRVTs entraram na lista de doenças ocupacionais do Ministério da Saúde. Isso reforça a importância de considerar as queixas e hábitos que levam à instalação de um DVRT, visando, principalmente, a sua prevenção. 
A partir do exposto, o objetivo deste estudo foi verificar e associar as características desfavoráveis à produção da voz no ambiente de trabalho, os hábitos vocais incorretos e as queixas vocais de profissionais e futuros profissionais da voz.

\section{Metodologia}

Estudo transversal, não-experimental, retrospectivo e quantitativo, com a utilização de dados de prontuários de uma clínica-escola de Fonoaudiologia, aprovado pelo Comitê de Ética em Pesquisa da instituição de origem (nº 4.338.295) (Pereira, Shitsuka, Parreira \& Shitsuka, 2018).

A população-alvo foi de profissionais e futuros profissionais da voz. São considerados profissionais da voz aqueles que dependem de sua voz para o sucesso profissional (Andrews, 2009; Andriollo, Bresolin et al., 2020; Brasil, 2018; Siqueira, Bastilha et al., 2016; Vilanova, Marques et al., 2016).

Para a seleção da amostra, foram adotados como critérios de inclusão: registro dos dados dos sujeitos profissionais ou futuros profissionais da voz nos prontuários, sexo masculino e feminino, faixa etária adulta entre 19 e 44 anos (MeSH/DeCS, 2020), assinatura do Termo de Consentimento Livre e Esclarecido da clínica-escola no prontuário. O critério de exclusão adotado foi o de prontuários com dados incompletos.

Desta forma, do total de 180 registros selecionados, 43 foram excluídos por apresentarem dados incompletos, resultando em uma amostra com os registros de 137 profissionais e futuros profissionais da voz, adultos, homens e mulheres, das seguintes profissões: estudantes que realizavam curso superior ou técnico que exigiria o uso profissional da voz falada e/ou cantada para atuação, professores, agentes de portaria, músicos, radialistas, recepcionistas, garçons, promotores de vendas, policiais, militares, porteiros, zeladores, atores e publicitários.

As informações verificadas nos 137 registros, extraídas do protocolo de anamnese utilizado na clínica-escola, relacionavam-se a fatores desfavoráveis à produção da voz no ambiente de trabalho (ar condicionado, ruído, poluição, ambiente que exige esforço vocal, mudanças frequentes de temperatura); aos hábitos vocais incorretos (falar muito ao telefone, falar forte, falar por muito tempo, gritar, etilismo, tabagismo, uso de drogas); e às queixas vocais (rouquidão, perda da eficiência vocal, mudanças súbitas na voz, cansaço ao falar, dores no pescoço, ardência, dor, ressecamento, sensação de estrangulamento ou de aperto na garganta).

Os dados, digitalizados em programa Excel, foram separados por grupos de fatores analisados. Em seguida, procedeuse à análise estatística descritiva dos grupos de variáveis e foram feitas tabelas com valores de frequência absoluta e relativa para descrever as características desfavoráveis à produção da voz no ambiente de trabalho, hábitos e queixas vocais.

Para a análise da proporção de cada variável e relação entre as variáveis categóricas, utilizou-se o Teste de Hipótese para Proporção, através da distribuição de probabilidade Binomial, o Teste Qui-quadrado de Pearson e o Teste Exato de Fisher. O nível de significância adotado para os testes estatísticos foi de $5 \%(\mathrm{p}<0,05)$.

\section{Resultados}

Na Tabela 1, verifica-se a frequência das variáveis analisadas com presença estatisticamente significativa de fatores desfavoráveis à produção da voz no ambiente de trabalho, hábitos vocais incorretos e queixas vocais. 
Tabela 1 - Frequência dos fatores desfavoráveis à produção da voz no ambiente de trabalho, hábitos vocais incorretos e queixas vocais

\section{$\operatorname{Sim~n~(\% )~Não~n~}(\%) \quad$ p-valor}

\begin{tabular}{|c|c|c|c|}
\hline Fatores desfavoráveis à produção da voz no ambiente de trabalho & $116(84,67)$ & $21(15,33)$ & $0,001 *$ \\
\hline Hábitos vocais incorretos & $132(96,35)$ & $5(3,65)$ & $0,001 *$ \\
\hline Queixas vocais & $92(67,15)$ & $45(32,85)$ & $0,001 *$ \\
\hline
\end{tabular}

Teste de Hipótese para Proporção p $<0,05$

* valores estatisticamente significativos

Fonte: Autores

A Tabela 2 apresenta a associação entre fatores desfavoráveis à produção da voz no ambiente de trabalho, hábitos vocais incorretos e queixas vocais com associação significativa entre os fatores desfavoráveis à produção da voz no ambiente de trabalho e os hábitos vocais incorretos e queixas vocais.

Tabela 2 - Associação entre fatores desfavoráveis à produção da voz no ambiente de trabalho, hábitos vocais incorretos e queixas vocais

Fatores desfavoráveis à produção da voz no ambiente de trabalho

p-valor

\begin{tabular}{|c|c|c|c|c|}
\hline & & Não n (\%) & $\operatorname{Sim} n(\%)$ & \multirow{3}{*}{$0,026^{*}$} \\
\hline \multirow{3}{*}{ Hábitos vocais incorretos } & Não n (\%) & $3(14,29)$ & $2(1,72)$ & \\
\hline & Sim n (\%) & $18(85,71)$ & $114(98,28)$ & \\
\hline & Não n (\%) & $21(100)$ & $38(32,76)$ & $0,001 *$ \\
\hline Queixas vocais & Sim n $(\%)$ & 0 & $78(67,24)$ & \\
\hline
\end{tabular}

Teste Exato de Fisher p<0,05; Teste Qui-quadrado $\mathrm{p}<0,05$

* valores estatisticamente significativos

Fonte: Autores

\section{Discussão}

A importância da investigação sobre a saúde, ambiente de trabalho e queixas vocais de profissionais da voz vêm sendo destacada por alguns estudos (Abou-Rafée, Zambon et al., 2018; Amaral, Zambon, Moreti, \& Behlau, 2017; Andriollo, Bresolin et al., 2020; Assad, Gama, Santos, \& Magalhães, 2017; Behlau, Zambon, \& Madazio 2014; Christmann, Cielo, Scapini, F.,..\& Bastilha, 2017; Kraemer, Moreira et al., 2020; Servilha \& Ruela, 2010), visando à elaboração de políticas públicas a fim de proteger esses trabalhadores. No entanto, poucos estudos referem-se às questões relativas a futuros profissionais da voz, abordando hábitos e queixas vocais que esses indivíduos apresentam antes mesmo de iniciar suas carreiras (Cielo, Ribeiro et al., 2015; Siqueira, Bastilha et al., 2016). O conhecimento sobre as condições favoráveis e desfavoráveis à produção vocal no ambiente de trabalho e sobre os hábitos vocais incorretos que levam a uma disfonia é fundamental para prevenir problemas vocais decorrentes do uso vocal profissional tanto dos profissionais quanto dos futuros profissionais.

No grupo de profissionais e futuros profissionais da voz analisado neste estudo, observou-se frequência significativa 
de fatores desfavoráveis à produção da voz no ambiente de trabalho, de hábitos vocais incorretos e de queixas vocais (Tabela 1). Houve, ainda, associação significativa entre fatores desfavoráveis à produção da voz no ambiente de trabalho e hábitos vocais incorretos, e entre fatores desfavoráveis à produção da voz no ambiente de trabalho e queixas vocais (Tabela 2). Esses resultados estão condizentes com aqueles encontrados em vários outros estudos com diferentes categorias de profissionais e futuros profissionais da voz, como relatado a seguir.

Recentemente, em revisão integrativa da literatura sobre a associação entre trabalho e distúrbio de voz, foram analisados 47 artigos sobre o tema. Os autores concluíram, através da análise desses estudos, que trabalhadores que usavam a voz profissionalmente apresentaram, com consistência, maior frequência de um DVRT. Os achados provêm de estudos transversais que fortalecem o nexo entre distúrbio de voz e trabalho e confirmam a alta prevalência desse agravo, especialmente em professores do ensino fundamental, de meia idade e de ambos os sexos. O DVRT associou-se fundamentalmente a fatores do ambiente e da organização do trabalho, especialmente ao ruído e à extensa carga horária, como também a fatores predisponentes concausais, a exemplo de alergias (Jesus, Ferrite et al., 2020).

Pesquisa caracterizou os sintomas vocais de futuros profissionais da voz, homens e mulheres, e verificou alta média de sintomas vocais, salientando-se a tosse ou pigarro e a dificuldade para falar em locais barulhentos, diretamente relacionados a usos vocais incorretos. Os autores concluíram que, com o aumento da demanda vocal profissional, esses indivíduos poderão ser considerados de risco para o desenvolvimento de distúrbios vocais (Cielo, Ribeiro et al., 2015).

Estudos realizados com docentes apresentam relatos de ruído elevado/insuportável na sala de aula e fora dela (Amaral, Zambon et al., 2017; Giannini, Latorre, \& Ferreira, 2013; Jardim, Barreto, \& Assunção, 2007; Marçal \& Peres, 2011; Pascotini, Ribeiro, \& Cielo, 2015; Valente, Botelho, \& Silva, 2015). Outras pesquisas constataram indicação de mais fatores negativos do que positivos em relação à saúde e à voz no ambiente de trabalho, estando condizente com os aspectos analisados no presente estudo como: ar condicionado, altos níveis de ruído externo, ambiente muito quente ou muito frio e poeirento (Abou-Rafée, Zambon et al., 2018; Amaral, Zambon et al., 2017; Andriollo, Bresolin et al., 2020; Christmann, Cielo et al., 2017; Giannini, Latorre et al., 2013; Jardim, Barreto et al., 2007; Marçal \& Peres, 2011; Pascotini, Ribeiro et al., 2015; Souza, Martins, Marcolino, Barbosa, Tamanini, \& Fonseca, 2015; Valente, Botelho et al., 2015; Vilanova, Marques et al., 2016). Ainda, um levantamento com professores (Marçal \& Peres, 2011) indicou a presença de ruído insuportável em sala de aula e dentro da escola, e a presença de poeira em sala de aula, além de fatores relacionados à carga horária e relações com colegas e alunos como fatores desfavoráveis à produção da voz no ambiente de trabalho.

Pesquisa destaca fatores prejudiciais à voz profissional de atores de teatro, que possuem alto risco de desenvolver problemas vocais devido às condições de trabalho e às características da profissão. Entre os fatores prejudiciais, encontram-se: dificuldades com uso da voz em cena; dificuldades relativas à articulação, respiração, qualidade da voz e projeção; exposição à ambiente de trabalho inadequado para a saúde vocal; alta demanda vocal decorrente da própria atividade teatral; e condições acústicas ambientais inadequadas, com ausência de amplificação sonora, gerando necessidade de aumento da loudness (Vilanova, Marques et al., 2016).

Estudo realizado com estudantes de comunicação (Cielo, Morisso, \& Conterno, 2009) encontrou percentual estatisticamente significativo em relação ao hábito de falar muito e o cansaço vocal como um dos sintomas mais referidos. Outros estudos relacionaram a queixa de fadiga vocal com os hábitos de falar forte e rápido, além do uso constante da voz (Abou-Rafée, Zambon et al., 2018; Assad, Gama et al., 2017; Christmann, Cielo et al., 2017). Questionários aplicados com cantores de rock (Gonçalvez, Amin, \& Behlau, 2010) mostraram que metade da amostra referiu alguma queixa vocal, sendo as mais citadas: rouquidão, tessitura limitada e falha na mudança de registro vocal.

Quanto aos hábitos relacionados à voz, pesquisa realizada com teleoperadores revelou que os mais relatados foram: falar muito $(69 \%)$, comer chocolate $(65 \%)$, tomar café $(60 \%)$. Dentre as queixas vocais mais mencionadas estavam: garganta 
seca $(53 \%)$, pigarro (33\%), cansaço ao falar (31\%). Esses profissionais acreditavam que os sintomas estavam relacionados ao uso intenso da voz, presença de ar-condicionado e ambiente de trabalho quente ou frio demais. Na comparação entre os grupos de teleoperadores satisfeitos e insatisfeitos com a voz, mostraram-se significativos a favor dos insatisfeitos: ter maior carga horária semanal; presença de distúrbios de vias aéreas como rinite e sinusite; hábito de falar muito; sintomas como cansaço ao falar, ardência na garganta, falhas na voz; relacionamento ruim; e estresse no trabalho (Ferreira, Akutsu, Luciano, \& Viviano, 2008). Tais achados convergem com os encontrados no presente estudo (Tabelas 1 e 2).

Em pesquisa com professores de uma rede municipal de ensino (Caporossi \& Ferreira, 2011), os hábitos citados que poderiam interferir de forma negativa na produção da voz foram: falar muito, falar em lugar aberto, gritar, fumar, falar carregando peso, e ingerir bebida alcoólica. Houve, ainda, associação significativa entre queixas vocais (autorreferidas) e hábitos vocais incorretos (falar muito). Esses resultados concordam com nosso estudo e com diversos outros (Amaral, Zambon et al., 2017; Bastos \& Hermes, 2018; Christmann, Cielo et al., 2017; Giannini, Latorre et al., 2013; Marçal \& Peres, 2011; Pascotini, Ribeiro et al., 2015; Valente, Botelho et al., 2015).

Os sintomas vocais mais encontrados em um grupo de professores foram rouquidão, dor de garganta, fazer força para falar, desaparecimento da voz, falhas na voz e voz grossa (Servilha \& Pereira, 2008). Os autores constataram que os fatores presentes no ambiente de trabalho e em sua organização podem levar à prejuízos na saúde geral e vocal dos professores. O trabalho de Petroucic e Friedman (2006) realizado com 12 indivíduos, sendo elas profissionais e não profissionais da voz com e sem queixas vocais, relatou que a maioria associou a perda de voz a problemas no trabalho. Entre docentes analisados (Jardim, Barreto et al., 2007), mais da metade da amostra referiu cansaço ao falar (61\%) e piora da qualidade vocal (56\%) nas últimas duas semanas, e aproximadamente metade das professoras relatou ruído elevado/insuportável na sala de aula (51\%).

Estudo recente avaliou os riscos ergonômicos e sua relação com a dor musculoesquelética em docentes, e observou que a maioria referiu pouco conhecimento sobre ergonomia e a sua não aplicação no dia a dia. Além disso, todos os professores apresentaram dor nos últimos 12 meses, e as regiões prevalentes foram a coluna lombar, o pescoço e os ombros. A partir dos resultados, os autores reforçaram a importância de que ocorram adaptações ergonômicas e mudança nos hábitos dos docentes para prevenir problemas de saúde e melhorar a qualidade de vida e do ensino (Kraemer, Moreira et al., 2020).

A partir desta discussão, verifica-se que a maioria dos trabalhos científicos consultados converge com os resultados da presente pesquisa em relação à presença e associação entre queixas/sintomas vocais e características do ambiente de trabalho desfavoráveis à produção da voz (Tabelas 1 e 2). Porém, em pesquisa com docentes, em 47,6\% deles a alteração vocal autorreferida não apresentou associação com características organizacionais do trabalho (Marçal \& Peres, 2011), o que difere de nosso trabalho (Tabela 2).

Em relação aos hábitos prejudiciais à saúde da voz, pesquisa realizada com cantores líricos e populares revelou que a maior parte dos cantores referiu nunca ingerir bebidas alcoólicas e não fumar ou fazer uso de outras drogas (Dassie-Leite, Duprat, \& Busch, 2011). Estudo com professores (Servilha \& Pereira, 2008) encontrou percentuais de 23,8\% de tabagismo, 9,5\% de etilismo, além do hábito de gritar e pigarrear (14,3\%), resultados que divergem dos nossos. Em nosso estudo, observamos valores percentuais de hábitos vocais incorretos significativamente altos (Tabela 1), divergindo dessas pesquisas.

Em outra investigação, o objetivo foi caracterizar e relacionar a quantidade e condições de hidratação, o tempo diário de uso vocal e as queixas de ressecamento de profissionais e futuros profissionais da voz. Verificou-se que a maioria deles utilizava a voz por tempo inferior a oito horas diárias e possuía água disponível no ambiente de trabalho, não havendo significância em relação à queixa de ressecamento. Ressalta-se a necessidade de fácil acesso à água no ambiente de trabalho para que o profissional tenha o hábito de hidratação como fator preventivo de disfonias (Siqueira, Bastilha et al., 2016).

Com base nos achados desta pesquisa e na literatura consultada, destaca-se a necessidade de alerta das organizações públicas e privadas que empregam profissionais da voz para as condições dos ambientes de trabalho, muitas vezes 
desfavoráveis à saúde vocal e global dos seus trabalhadores. Com um ambiente de trabalho favorável, o desenvolvimento das tarefas torna-se mais eficiente, contribuindo para o melhor andamento da empresa ou instituição, diminuindo gastos referentes a licenças por problemas de saúde e indenizações.

O trabalho de orientação vocal mostra-se de suma importância para a diminuição de hábitos vocais incorretos, minimizando as queixas vocais. Salienta-se que o trabalho fonoaudiológico preventivo, associado a melhores condições de trabalho, pode resultar em diminuição significativa da prevalência de disfonia em profissionais da voz, levando à diminuição dos gastos públicos relacionados com o seu tratamento.

Uma das limitações deste trabalho foi a impossibilidade de obter um número equitativo de sujeitos nas diferentes categorias de profissionais e futuros profissionais da voz que caracterizaram a amostra. Outra limitação foi a amostra não representar todas as possíveis categorias profissionais da voz. Novas pesquisas são necessárias com maior abrangência de categorias de profissionais e futuros profissionais da voz, considerando que, quanto maior for o conhecimento a respeito das queixas e hábitos vocais mais comuns entre os profissionais e futuros profissionais da voz, maiores serão as chances de prevenção e tratamento precoce dos DVRT de forma mais efetiva com base em suas características específicas.

\section{Conclusão}

Neste grupo de profissionais e futuros profissionais da voz, verificou-se alta ocorrência de fatores desfavoráveis à produção da voz no ambiente de trabalho, queixas vocais e hábitos vocais incorretos, bem como associação entre as queixas vocais e hábitos vocais incorretos e os fatores desfavoráveis à produção da voz no ambiente de trabalho. Portanto, destaca-se a que a prevenção, somada a melhores condições de trabalho, pode resultar em diminuição dos quadros de disfonia em profissionais e futuros profissionais da voz.

Esses resultados servem de alerta e podem ser utilizados para monitorar e prevenir alterações vocais em profissionais e futuros profissionais da voz, sempre que possível antes da instalação e possíveis consequências de um DVRT. Portanto, sugere-se a realização de mais pesquisas com diferentes categorias de profissionais e futuros profissionais da voz, visando ampliar o conhecimento sobre os aspectos vocais e o ambiente de trabalho e as possibilidades de atuação fonoaudiológica.

\section{Agradecimentos}

O presente trabalho foi realizado com apoio da Coordenação de Aperfeiçoamento de Pessoal de Nível Superior Brasil (CAPES) - Código de Financiamento 001 e Conselho Nacional de Desenvolvimento Científico e Tecnológico (CNPQ) (Grant 301326/2017-7).

\section{Referências}

Abou-Rafée, M., Zambon, F., Badaró, F., \& Behlau, M. (2019).Vocal fatigue in dysphonic teachers who seek treatment. CoDAS, 31(3), 1-6. 10.1590/2317$1782 / 20182018120$

Amaral, A. C., Zambon, F., Moreti, F., \& Behlau, M. (2017). Desconforto do trato vocal em professores após atividade letiva. CoDAS, $29(2)$, e20160045. $10.1590 / 2317-1782 / 20172016045$

Andrade, S. R., Cielo, C. A., Schwarz, K., \& Ribeiro, V. V. (2016). Vocal therapy and nasal sounds: effects on Hyperfunctional dysphonia. Revista CEFAC, 18(1), 263-272. 10.1590/1982-021620161810115

Andrews, M. L. Distúrbios em adultos e seu tratamento. In: Andrews, M.L. (2009). Manual de tratamento da voz: da pediatria à geriatria: Cenage Learning.

Andriollo, D. B., Bresolin, F. A., Frigo, L. F., \& Cielo, C. A. (2020). Treinamento fisioterapêutico intensivo do centro de força corporal: estudo de uma profissional da voz. Research, Society and Development, 9(3), e146932550. 10.33448/rsd-v9i3.2550.

Assad, J. P., Gama, A. C. C., Santos, J. N., \& Magalhães, M. C. (2017). The effects of amplification on vocal dose in teachers with dysphonia. Journal of Voice, 33(1), 73-79. 10.1016/j.jvoice.2017.09.011

Behlau, M., Zambon, F., \& Madazio, G. (2014). Gerenciando disfonia em usuários de voz ocupacional. Current Opinion in Otolaryngology \& Head and Neck Surgery, 22(3), 188-94. 10.1097/MOO.0000000000000047 
Branco, J. C., Silva, F. G., Jansen, K., \& Giusti, P. H. (2011). Prevalência de sintomas osteomusculares em professores de escolas públicas e privadas do ensino fundamental. Fisioterapia e Movimento, 24(2), 307-314. 10.1590/S0103-51502011000200012

Caporossi, C., \& Ferreira, L. P. (2011). Sintomas vocais e fatores relativos ao estilo de vida em professores. Revista CEFAC, 13(1),132-139. 10.1590/S151618462010005000099

Christmann, M. K., Cielo, C. A., Scapini, F., \& Bastilha, G. R. (2017). Controlled and randomized clinical trial of intensive shortterm voice therapy with finger kazzo technique in teachers. Audiology-Communication Research, 22(e1791): 1-12. 10.1590/2317-6431-2016-1791

Cielo, C. A., Morisso, M. F., \& Conterno, G. (2009). Hábitos e queixas vocais de estudantes de comunicação. Salusvita, 28(2),169-181. http://iah.iec.pa.gov.br/iah/fulltext/lilacs/salusvita/2009v28n2/salusvita2009v28n2p169-181.pdf

Cielo, C. A., Christmann, M. K., Ribeiro, V. V., Hoffmann, C. F., Padilha, J. F., Steidl, E. M. S., \& Frigo, L. F. (2014). Musculoskeletal stress syndrome, extrinsic laryngeal muscles and body posture: theoretical considerations. Revista CEFAC, 16(5), 1639-1649. 10.1590/1982-0216201410613

Cielo, C. A., Ribeiro, V. V., \& Bastilha, G. R. (2015). Medidas vocais espectrográficas, queixas vocais e dados ocupacionais de professoras do ensino fundamental. Distúrbios da Comunicação, 27(1), 129-137. https://revistas.pucsp.br/index.php/dic/article/view/19582/16986

Cielo, C. A., Ribeiro, V. V., \& Hoffmann, C. F. (2015). Sintomas vocais de futuros profissionais da voz. Revista CEFAC, 17(1), 34-43. 10.1590/19820216201517013

Dassie-Leite, A. P., Duprat, A. C., \& Busch, R. (2011). Comparação de hábitos de bem estar vocal entre cantores líricos e populares. Revista CEFAC, 13(1), 123-131. 10.1590/S1516-18462010005000118

Ferreira, L. P., Akutsu, C. M., Luciano, P. \& Viviano, N. A. G. (2008). Condições de produção vocal de teleoperadores: correlação entre questões de saúde, hábitos e sintomas vocais. Revista Sociedade Brasileira de Fonoaudiologia. 13(4), 307-315. 10.1590/S1516-80342008000400003

Giannini, S. P. P., Latorre, M. R. D. O., \& Ferreira, L. P. (2013). Distúrbio de voz relacionado ao trabalho docente: um estudo caso-controle. CoDAS, 25(6), 566-576. 10.1590/S0102-311X2012001100011

Gonçalves, A., Amin, E., \& Behlau, M. (2010). Análise do grau global e tensão da voz em cantores de roque. Pró-Fono Revista de Atualização Científica, 22(3), 195-200. 10.1590/S0104-56872010000300007

Jardim, R., Barreto, S. M., \& Assunção, A. (2007). Condições de trabalho, qualidade de vida e disfonia entre docentes. Cad. Saúde Pública, 23(10), 24392461. 10.1590/S0102-311X2007001000019

Jesus, M. T., Ferrite, S., Araújo, T. M., \& Masson, M. L. V. (2020). Distúrbio de voz relacionado ao trabalho: revisão integrativa. Revista Brasileira de Saúde Ocupacional, 45:e26, 1-14. 10.1590/2317-6369000040218

Karakaya, I. C., Karakaya, M. G., Tunç, E., \& Kihtir, M. (2015). Musculoskeletal Problems and Quality of Life of Elementary School Teachers, International Journal of Occupational Safety and Ergonomics. International Journal of Occupational Safety and Ergonomics, 21(3), 344-50. $10.1080 / 10803548.2015 .1035921$

Kraemer, K., Moreira, M. F., \& Guimarães, B. (2020). Musculoskeletal pain and ergonomic risks in teachers of a federal institution. Revista Brasileira de Medicina do Trabalho, 18(3), 343-351. 10.47626/1679-4435-2020-608

Mango, M. S. M., Carilho, M. K., Drabovski, B., Joucoski, E., Garcia, M. C., \& Gomes, A. R. S. (2012). Análise dos sintomas osteomusculares de professores do ensino fundamental em Matinhos (PR). Fisioterapia e Movimento, 25(4), 785-794. 10.1590/S0103-51502012000400011

Marçal, C. C. B, \& Peres, M. P. (2011). Self-reported voice problems among teachers: prevalence and associated factors. Revista de Saúde Pública, 45(3), 1-8. $10.1590 /$ S0034-89102011005000025

Ministério da Saúde. (2018). Distúrbio de Voz Relacionado ao Trabalho - DVRT. Ministério da Saúde, Secretaria de Vigilância em Saúde, Departamento de Vigilância em Saúde Ambiental e Saúde do Trabalhador. - Brasília: Ministério da Saúde, 2018.42 p. http://bvsms.saude.gov.br/bvs/publicacoes/disturbio_voz_relacionado_trabalho_dvrt.pdf

Pascotini, F., Ribeiro, V. V., \& Cielo, C. A. (2015). Voz de professoras do ensino fundamental com queixas vocais de diferentes redes de ensino. Distúrbios da Comunicação, 27(1), 138-150. https://revistas.pucsp.br/index.php/dic/article/view/18873/16334

Pereira, A. S., Shitsuka, D. M., Parreira, F. J., \& Shitsuka, R. (2018). Metodologia da pesquisa científica. [e-book]. Santa Maria: Ed. UAB/NTE/UFSM. https://repositorio.ufsm.br/bitstream/handle/1/15824/Lic_Computacao_Metodologia-Pesquisa-Cientifica.pdf?sequence=1

Pinho, S. M. R. (2007). Manual de higiene vocal para profissionais da voz. (4a ed.): Pró-fono.

Servilha, E. A. M., \& Ruela, I. S. (2010). Riscos ocupacionais à saúde e voz de professores: especificidades das unidades de rede municipal de ensino. Revista CEFAC, 12(1), 109-114. 10.1590/S1516-18462009005000061

Siqueira, M. A., Bastilha, G. R., Lima, J. P. M., \& Cielo, C. A. (2016). Hidratação vocal em profissionais e futuros profissionais da voz. Revista CEFAC, 18(4), 908-914. 10.1590/1982-0216201618417415

Souza, D. B. O., Martins, L. V., Marcolino, A. M., Barbosa, R. I., Tamanini, G., Fonseca, M. C. R. (2015). Capacidade para o trabalho e sintomas osteomusculares em trabalhadores de um hospital público. Revista Fisioterapia e Pesquisa, 22(2), 182-190. 10.590/1809-2950/14123722022015

Valente, A. M. S. L., Botelho, C., Silva, A. M. C. (2015). Distúrbio de voz e fatores associados em professors da rede pública. Revista Brasileira de Saúde Ocupacional, 40(132), 183-195. 10.1590/0303-7657000093814 
Research, Society and Development, v. 10, n. 2, e53110212531, 2021

(CC BY 4.0) | ISSN 2525-3409 | DOI: http://dx.doi.org/10.33448/rsd-v10i2.12531

Vilanova, J. R., Marques, J. M., Ribeiro, V. V., Oliveira, A. G., Teles, L., \& Silverio, K. C. A. (2016). Atores profissionais e estudantes de teatro: aspectos vocais relacionados à prática. Revista CEFAC, 18(4), 897-907. 10.1590/1982-021620161849315 